\title{
Escape Beyond Vermilion Border
}

National Cancer Institute

\section{Source}

National Cancer Institute. Escape Beyond Vermilion Border. NCI Thesaurus. Code

C127186.

A finding of escape beyond the vermilion border during swallowing. 